\title{
Ethyl acetate extract of Hypericum japonicum induces apoptosis via the mitochondria-dependent pathway in vivo and in vitro
}

\author{
QUNCHUAN ZHUANG ${ }^{1,2}$, JING LI $^{3}$, YOUQIN CHEN ${ }^{4,5}$, JIUMAO LIN $^{1,2}$, FAZE LAI $^{6}$, \\ XUZHENG CHEN ${ }^{1,2}$, XINDENG LIN $^{6}$ and JUN PENG ${ }^{1,2,5}$ \\ ${ }^{1}$ Academy of Integrative Medicine and ${ }^{2}$ Fujian Key Laboratory of Integrative Medicine on Geriatrics, \\ Fujian University of Traditional Chinese Medicine, Fuzhou, Fujian 350122; ${ }^{3}$ Department of Medical Technology, \\ Fujian Health College, Fuzhou, Fujian 350101, P.R. China; ${ }^{4}$ Rainbow Babies and Children's Hospital, \\ Case Western Reserve University School of Medicine, Cleveland, OH 44106, USA; \\ ${ }^{5}$ Postdoctoral Workstation, Zhangzhou Pien Tze Huang Pharmaceutical Co., Ltd., Zhangzhou, Fujian 363000; \\ ${ }^{6}$ Department of Pharmacology, Fujian University of Traditional Chinese Medicine, Fuzhou, Fujian 350108, P.R. China
}

Received February 25, 2015; Accepted May 19, 2015

DOI: $10.3892 / \mathrm{mmr} .2015 .4086$

\begin{abstract}
The widely-used Chinese medicinal herb Hypericum japonicum, also known as Hypericum japonicum Thunb or Tianjihuang, displays potent anti-carcinogenic effects against liver cancer. However, the molecular mechanism underlying the therapeutic effects of Hypericum japonicum remains to be elucidated. The present study investigated the in vivo efficacy of ethyl acetate extract of Hypericum japonicum (EAEHJ) against tumor growth in an H22 cell-bearing liver cancer mouse model. Treatment with EAEHJ significantly reduced tumor weight, but had no effect on murine body weight. The results of the present study also showed that EAEHJ induced $\mathrm{H} 22$ cell apoptosis in vivo. In addition, the anti-carcinogenic effects of EAEHJ were investigated in vitro. The results of the present study demonstrate that both phospholipid asymmetry in the plasma membrane and mitochondrial membrane potential were deregulated in HepG2 human hepatoma cells, following treatment with EAEHJ. Treatment with EAEHJ also increased the ratio of pro-apoptotic B-cell lymphoma 2 (Bcl-2)-associated $\mathrm{X}$ protein (Bax) to anti-apoptotic $\mathrm{Bcl}-2$, and activated the caspase- 9 signaling pathway. These results suggest that EAEHJ is able to trigger the apoptosis of liver cancer cells via the mitochondria-dependent pathway.
\end{abstract}

Correspondence to: Professor Jun Peng, Fujian University of Traditional Chinese Medicine, 1 Huatuo Road, Fuzhou, Fujian 350122, P.R. China

E-mail: pjunlab@hotmail.com

Key words: apoptosis, liver cancer, HepG2 cells, herbal medicine, mitochondria, Hypericum japonicum

\section{Introduction}

Hepatocellular carcinoma (HCC) is the fifth most frequently diagnosed cancer in men, and the second leading cause of cancer-associated mortality worldwide (1). In 2008, an estimated 748,300 new HCC cases and 695,900 HCC fatalities occurred worldwide, half of which were located in China $(1,2)$. Although substantial progress has been made in the chemotherapeutic treatment of HCC, the efficacy of chemotherapeutic drugs is often hampered by various adverse side effects. Therefore, natural products have become increasingly important for the discovery of novel pharmaceutical treatment strategies. Traditional Chinese herbology pioneers the use of natural products in the biomedical field, and Chinese medicinal herbs are increasingly being used for their antitumor effects.

Hypericum japonicum, also know as Tianjihuang, is a Chinese medicinal herb which has been used for centuries in the treatment of infectious hepatitis, bacterial infection, and tumors (3-5). Hypericum japonicum has been shown to exhibit significant anti-carcinogenic effects in clinical settings, specifically with regards to tumors of the digestive system, such as HCC. In addition, treatment with Hypericum japonicum has been shown to increase patient survival and quality of life, and decrease tumor size (6). Furthermore, previous studies demonstrated that Hypericum japonicum exhibited hepatoprotective effects on $\mathrm{CCL}_{4}$ - and acetaminophen-induced acute hepatotoxicity in rat models $(3,7)$. However, the mechanism underlying the hepatoprotective effects of Hypericum japonicum on HCC remains to be elucidated.

The present study evaluated the in vivo efficacy of ethyl acetate extract of Hypericum japonicum (EAEHJ) against tumor growth in an $\mathrm{H} 22$ cell-bearing liver cancer mouse model, and investigated the molecular mechanisms underlying the anti-carcinogenic effects of EAEHJ in vitro. It is hypothesized that the anti-carcinogenic effects of Hypericum japonicum in vitro likely result from its ability to promote apoptosis of cancer cells via the mitochondria-dependent pathway. 


\section{Materials and methods}

Materials and reagents. Dulbecco's modified Eagle's medium (DMEM), trypsin-EDTA, and penicillin-streptomycin were purchased from Thermo Fisher Scientific, Inc. (Waltham, MA, USA). Fetal bovine serum (FBS), TRIzol ${ }^{\circledR}$ Reagent, JC-1, and Caspase-3 and -9 Colorimetric Protease Assay kits were purchased from Invitrogen Life Technologies (Carlsbad, CA, USA). SuperScript II reverse transcriptase was purchased from Thermo Fisher Scientific, Inc. The B-cell lymphoma 2 (Bcl-2; \#2872; 1:1,000), Bcl-2-associated X protein (Bax; \#2772; $1: 1,000)$ and $\beta$-actin $(\# 4967 ; 1: 1,000)$ primary antibodies, and the horseradish peroxidase (HRP)-conjugated secondary antibodies $(\# 7075 ; 1: 2,000)$ were purchased from Cell Signaling Technology, Inc. (Danvers, MA, USA). The fluorescein isothiocyanate (FITC)-conjugated Annexin V Apoptosis Detection kit was purchased from BD Biosciences (San Jose, CA, USA). All the other reagents, unless otherwise stated, were obtained from Sigma-Aldrich (St. Louis, MO, USA).

EAEHJ preparation. A total of $200 \mathrm{~g}$ Hypericum japonicum was extracted from Hypericum japonicum Thunb. ex Murray herbs (Fujian Tongchun Pharmaceutical Co., Ltd., Fuzhou, China) using $1.6195 \%$ ethanol, according to the reflux method (8), prior to being filtered. The ethanol was then evaporated using a RE-2000 Rotary Evaporator (Shanghai Yarong Biochemical Instrument Factory, Shanghai, China). The solution obtained following ethanol evaporation was concentrated in a rotary evaporator at $50^{\circ} \mathrm{C}$ until $26 \mathrm{~g}$ Hypericum japonicum ethanol extract powder was produced. A total of $20 \mathrm{~g}$ of Hypericum japonicum ethanol extract powder was then resuspended in $200 \mathrm{ml}$ distilled water. Four organic solvents in increasing order of polarity: Petroleum ether, chloroform, ethyl acetate, and butan-1-ol were subsequently partitioned using a separating funnel. The ethyl acetate extract was used for further experimentation, and was dissolved in dimethyl sulfoxide (DMSO) in order to form a $600 \mathrm{mg} / \mathrm{ml}$ EAEHJ stock solution, prior to being stored at $-20^{\circ} \mathrm{C}$. The experimental concentrations of EAEHJ $(0,0.5,0.75,1.5$ and $3 \mathrm{mg} / \mathrm{ml})$ were obtained by diluting the stock solution in culture medium supplemented with $<0.5 \%$ DMSO.

Animals. Male Kunming mice (10-12 weeks of age, weighing 18-22 g) were obtained from Vital River Laboratory Animal Technology Co., Ltd. (Beijing, China), and housed in a sterile humidified environment $(50-60 \%)$ at $22^{\circ} \mathrm{C}$, with a $12 \mathrm{~h}$ light/dark cycle. Food and water were provided ad libitum throughout the experiment. All animal treatments were conducted in strict accordance with international ethical guidelines, according to the National Institute of Health Care and Use of Laboratory Animals Guide (9), and all experiments were approved by the Institutional Animal Care and Use Committee of the Fujian University of Traditional Chinese Medicine (Fuzhou, China). The H22 liver cancer cells were injected subcutaneously into the mice, as previously described $(10,11)$. Briefly, the ascites $\mathrm{H} 22$ cells were harvested, diluted to a concentration of $5.0 \times 10^{6} / \mathrm{ml}$ using sterilized normal saline, and injected subcutaneously into the right axillary region of the mice. The tumor volumes were subsequently calculated using the following formula: Volume $=\pi / 6 \times \mathrm{L} \mathrm{x} \mathrm{W}^{2}(12,13)$. When the tumors reached a size of $100 \mathrm{~mm}^{3}$, the animals were randomly assigned into two groups $(\mathrm{n}=8)$, and intragastrically administered either $60 \mathrm{mg} / \mathrm{kg} / \mathrm{d}$ EAEHJ or saline for 10 days. At the end of the experiment, the animals were anaesthetized with intraperitoneal pelltobarbitalum natricum $(300 \mathrm{mg} / \mathrm{kg})$, and the tumor tissue samples were removed and weighed. The tumor tissue samples were subsequently fixed with $10 \%$ formaldehyde for $12 \mathrm{~h}$, and placed on paraffin-embedded tumor slides. The inhibitory effects of EAEHJ on tumor growth were evaluated by measuring the tumor growth inhibition rate. The tumor growth inhibition rates were calculated as follows: Tumor inhibition rate $=(1-$ average tumor weights of the treatment group/average tumor weight of control group) x 100 .

Cell culture and cell viability assay. The H22 murine hepatoma and HepG2 human hepatoma cell lines were obtained from the American Type Culture Collection (Manassas, VA, USA). Both cell lines were cultured in DMEM supplemented with $10 \%(\mathrm{v} / \mathrm{v}) \mathrm{FBS}, 100 \mathrm{U} / \mathrm{ml}$ penicillin and $100 \mu \mathrm{g} / \mathrm{ml}$ streptomycin at $37^{\circ} \mathrm{C}$ in in an atmosphere containing $5 \% \mathrm{CO}_{2}$. The cells were cultured to $80-90 \%$ confluence. The cells used throughout the present study were subjected to $<20$ cell passages. Cellular morphological changes were observed using a phase-contrast microscope (IX70; Olympus Corporation, Tokyo, Japan). The images were captured at a x100 magnification, and cell viability was assessed using an MTT colorimetric assay. Briefly, the HepG2 human hepatoma cells were seeded into 96-well plates at a density of $1.0 \times 10^{4}$ cells/well in $0.1 \mathrm{ml}$ medium. Following a period of $24 \mathrm{~h}$, the cells were treated with various concentrations $(0,0.5,0.75,1.5$ and $3 \mathrm{mg} / \mathrm{ml})$ of EAEHJ. Treatment with $0.5 \%$ DMSO was included as a vehicle control. At the end of the cellular treatment period, the medium was discarded, and $100 \mu \mathrm{l}$ MTT solution $[0.5 \mathrm{mg} / \mathrm{ml}$ in phosphate-buffered saline (PBS)] was added to each well. Following $4 \mathrm{~h}$ of incubation at $37^{\circ} \mathrm{C}$, MTT was discarded and $100 \mu \mathrm{l}$ DMSO was added to each well. The purple/blue MTT formazan precipitate was dissolved, and the absorbance was measured at $570 \mathrm{~nm}$ using an ELx800 ELISA reader (BioTek Instruments, Inc., Winooski, VT, USA).

In situ apoptosis detection by TUNEL staining. The $5 \mu \mathrm{m}$ tumor tissue sample sections were analyzed by TUNEL staining using a TumorTACS ${ }^{\mathrm{TM}}$ In Situ Apoptosis Detection kit in accordance with the manufacturer's instructions (R\&D Systems, Inc., Minneapolis, MN, USA). The apoptotic diaminobenzidine (DAB)-positive cells (brown stained) were counted in five arbitrarily selected microscopic fields at a x400 magnification (DM IL LED Microscope; Leica Microsystems $\mathrm{GmbH}$, Wetzlar, Germany).

Immunohistochemical analysis. The slides containing the tumor tissue samples were subjected to antigen retrieval with citrate buffer, and the endogenous peroxidase activity was blocked using 3\% hydrogen peroxide in water. For immunohistochemical staining, the slides were incubated with rabbit polyclonal antibodies targeting Bax (sc-6236) and Bcl-2 (sc-783) (1:200; Santa Cruz Biotechnology, Inc., Dallas, TX, USA). Following washing with PBS, the slides were incubated with biotinylated HRP-conjugated streptavidin secondary antibody 
(E0432; 1:1,000; Dako, Glostrup, Denmark), prior to being further washed with PBS. The slides were then incubated with DAB, prior to being counterstained with diluted Harris hematoxylin. Following staining, five x400 magnification fields (DM IL LED Microscope) were randomly selected in each slide, and the average ratio of positive cells in each field was counted using the Image-Pro Plus true color multi-functional cell image analysis management system, version 6.0 (Media Cybernetics, Inc., Rockville, MD, USA). In order to prevent any non-specific staining, PBS was used as a replacement to the primary antibody in the negative control.

Annexin V-FITC/propidium iodine (PI) staining apoptosis assay. Following incubation with various concentrations $(0,0.5,0.75$, 1.5 and $3 \mathrm{mg} / \mathrm{ml}$ ) of EAEHJ, the apoptotic levels of the HepG2 human hepatoma cells were determined by fluorescence-activated cell sorting (FACS) flow cytometry (FACSCalibur; BD Biosciences), using an Annexin V-FITC/PI kit (BD Biosciences). Staining was performed according to the manufacturer's instructions. The Annexin V/PI-double negative cells were considered to be viable. The Annexin V-positive/PI-negative population represents cells undergoing early apoptosis, while the population of Annexin V/PI double-positive cells represents cells in the late apoptotic stage.

Detection of $\Delta \Psi m$ by $J C-1$ staining. JC-1 is a cationic dye that exhibits potential-dependent accumulation in mitochondria, indicated by a shift in fluorescence emission from green to red. Therefore, JC-1 may be used as an indicator of mitochondrial potential. The HepG2 human hepatoma cells were plated in 6 -well plates at $1 \times 10^{5}$ cells/well in a volume of $2 \mathrm{ml}$, and incubated in DMEM overnight. The cells were then treated with various concentrations $(0,0.5,0.75,1.5$ and $3 \mathrm{mg} / \mathrm{ml})$ of EAEHJ for $24 \mathrm{~h}$, prior to being cultured in fresh media in the absence of FBS for a further $24 \mathrm{~h}$. The cells were washed twice with PBS, and resuspended in $1 \mathrm{ml}$ DMEM supplemented with $10 \% \mathrm{FBS}$ and $10 \mu \mathrm{g} / \mathrm{ml} \mathrm{JC}-1$, at $37^{\circ} \mathrm{C}$ in an atmosphere containing $5 \% \mathrm{CO}_{2}$ for $30 \mathrm{~min}$. Both red and green fluorescence emissions were analyzed by flow cytometry following JC-1 staining. The green and red fluorescence intensities, measured respectively at 520-530 nm (PMT2) and $590 \mathrm{~nm}$ (PMT3) for 1.0x104 individual cells, were analyzed by FACS analysis (BD Biosciences). The $\Delta \Psi \mathrm{m}$ values were expressed as the ratio of PMT3 to PMT2.

Reverse transcriptase-polymerase chain reaction (RT-PCR) and western blot analysis. A total of $2 \times 10^{5}$ HepG2 human hepatoma cells were seeded into 6-well plates in $2 \mathrm{ml}$ medium, and treated with various concentrations $(0,0.5,0.75,1.5$ and $3 \mathrm{mg} / \mathrm{ml}$ ) of EAEHJ for $24 \mathrm{~h}$. Total RNA from HepG2 human hepatoma cells was isolated using TRIzol ${ }^{\circledR}$ Reagent. A total of $1 \mu \mathrm{g}$ Oligo(dT)-primed RNA was reverse-transcribed using SuperScript II reverse transcriptase, according to the manufacturer's instructions. The obtained cDNA was used to determine the mRNA expression levels of Bcl-2 or Bax using Taq DNA polymerase (Thermo Fisher Scientific, Inc.). GAPDH was used as an internal control. The sequences of the primers used for Bcl-2, Bax, and GAPDH amplification were as follows: Bcl-2 forward, 5'-CAG CTG CAC CTG ACG CCC TT-3', and reverse, 5'-GCC TCC GTT ATC CTG GAT CC-3'; Bax forward, 5'-TGC TTC AGG GTT TCA TCC AGG-3', and reverse, 5'-TGG CAA
AGT AGA AAA GGG CGA-3'; and GAPDH forward, 5'-GT CAT CCA TGA CAA CTT TGG-3', and reverse, 5'-GA GCT TGA CAA AGT GGT CGT-3'. Cycling was conducted using the S1000 Thermal Cycler (Bio-Rad Laboratories, Inc., Hercules, CA, USA) with the following cycling conditions: Initial denaturation at $95^{\circ} \mathrm{C}$ for $5 \mathrm{~min}, 30 \mathrm{sec}$ rest, 30 cycles of annealing at a temperature $5^{\circ} \mathrm{C}$ below the melting point of the primers and extension at $72^{\circ} \mathrm{C}$ both for $\sim 1 \mathrm{~min} / \mathrm{kb}$ of expected product $10 \mathrm{~min}$ on the last cycle. PCR products were then analyzed by electrophoresis on a $2 \%$ agarose gel. The DNA bands were examined using a Gel Doc 2000 system (Bio-Rad Laboratories, Inc.). In order to carry out the western blot analysis, the HepG2 human hepatoma cells treated with EAEHJ were lysed using Mammalian Protein Extraction Reagent cell lysis buffer (Thermo Fisher Scientific, Inc.) containing protease (EMD Millipore, Billerica, MA, USA) and phosphatase inhibitor cocktails. The cell lysates were separated by $12 \%$ SDS-PAGE prior to immunoblotting to polyvinylidene fluoride (PVDF) membranes using the iBlot Western Detection Stack/iBlot Dry Blotting system (Invitrogen Life Technologies). The PVDF membranes were blocked using SuperBlock T20 Blocking Buffer (Thermo Fisher Scientific, Inc.) for $30 \mathrm{~min}$, and washed in tris-buffered saline containing $0.25 \%$ Tween ${ }^{\circledR} 20$ (TBST), prior to being incubated with the primary antibodies overnight at $4^{\circ} \mathrm{C}$. Following washing with TBST, the membranes were incubated with the HRP-conjugated secondary antibody for $1 \mathrm{~h}$ at room temperature. Finally, the blots were developed using Super Signal Pico Substrate (Thermo Fisher Scientific, Inc.), and images were captured using a Kodak Image Station 400R (Kodak, Rochester, NY, USA).

Analysis of caspase activation. Quantitative measurement of caspase activity was conducted using caspase-3 and -9 Colorimetric Protease Assay kits, according to the manufacturer's instructions. Briefly, following treatment with various concentrations $(0,0.5,0.75,1.5$ and $3 \mathrm{mg} / \mathrm{ml})$ of EAEHJ for $24 \mathrm{~h}$, the HepG2 human hepatoma cells were lysed with lysis buffer on ice for $30 \mathrm{~min}$. The lysed cells were centrifuged at $16,000 \mathrm{x} \mathrm{g}$ for $10 \mathrm{~min}$, and $100 \mu \mathrm{g}$ protein samples were incubated with the following $50 \mu \mathrm{l}$ colorimetric tetrapeptides: Asp-Glu-Val-Asp (DEVD)-p-nitroaniline (pNA) (specific substrate of caspase-3), or Leu-Glu-His-Asp (LEHD)-pNA (specific substrate of caspase-9), in total darkness at $37^{\circ} \mathrm{C}$, for $2 \mathrm{~h}$. Sample absorbance was measured at $405 \mathrm{~nm}$ using an ELx800 ELISA reader. The data were normalized to the activity levels of caspase-3 and -9 in the control cells treated with $0.5 \%$ DMSO vehicle, and represented as the "fold of control".

Statistical analysis. The data are expressed as the mean \pm standard deviation and were analyzed with SPSS software, version 13.0 (SPSS, Inc., Chicago, IL, USA). The statistical significance of each experimental test condition was assessed using an unpaired Student's t-test. $\mathrm{P}<0.05$ was considered to indicate a statistically significant difference.

\section{Results}

Inhibitory effects of EAEHJ in H22 cell-bearing mice, and Hep 2 human hepatoma cells. Following the sacrifice of the mice, the tumors were harvested and weighed. The tumor 
Table I. Inhibitory effects of Hypericum japonicum ethyl acetate extract (EAEHJ) in H22 cell-bearing liver cancer mice.

\begin{tabular}{|c|c|c|c|c|}
\hline \multirow[b]{2}{*}{ Group } & \multicolumn{2}{|c|}{ Body weight (g) } & \multirow[b]{2}{*}{ Tumor weight (g) } & \multirow[b]{2}{*}{ Tumor inhibition rate (\%) } \\
\hline & Pre-treatment & Post-treatment & & \\
\hline Control & $20.6 \pm 0.99$ & $27.4 \pm 1.29$ & $0.378 \pm 0.156$ & - \\
\hline EAEHJ & $21.7 \pm 0.97$ & $26.5 \pm 1.12$ & $0.230 \pm 0.098^{\mathrm{a}}$ & 39.2 \\
\hline
\end{tabular}
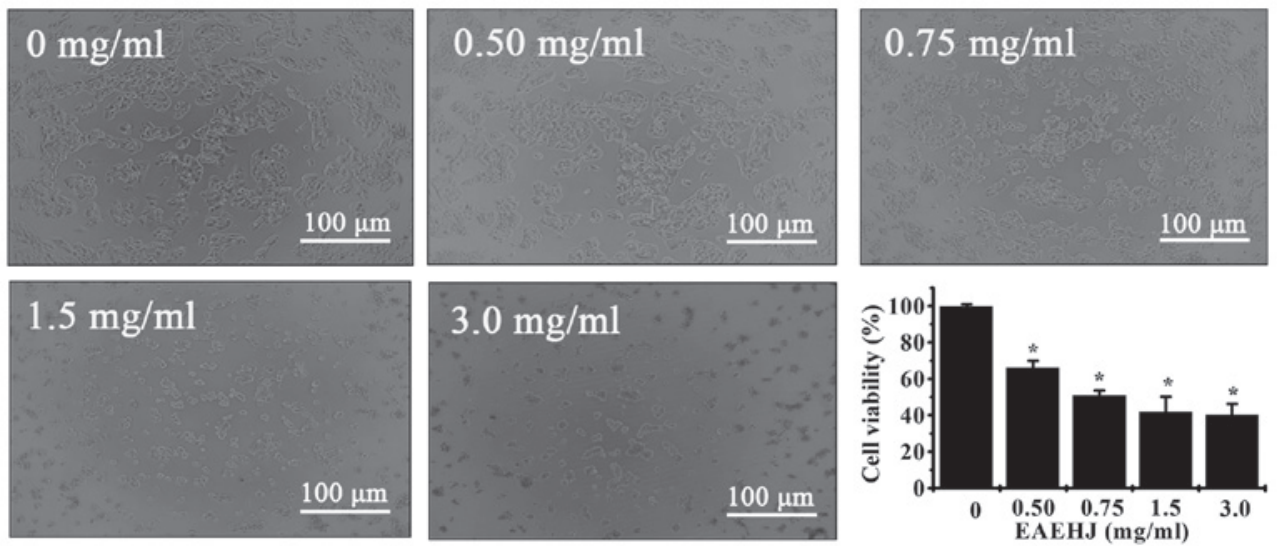

Figure 1. Effects of Hypericum japonicum ethyl acetate extract (EAEHJ) on cellular morphological changes and cell viability of HepG2 human hepatoma cells. The HepG2 human hepatoma cells were treated with various concentrations of EAEHJ for 24 h, and cellular morphological changes were subsequently observed using phase-contrast microscopy. The images were captured at a x100 magnification for each experiment performed in triplicate. Cell viability was assessed using an MTT assay. The MTT assay indicated that EAEHJ treatment significantly reduced HepG2 human hepatoma cell viability in a dose-dependent manner. The data are presented as the mean \pm standard deviation from three independent experiments. ${ }^{*} \mathrm{P}<0.01$, vs. the control cells $(100 \%$, treated with $0.5 \%$ dimethyl sulphoxide vehicle).

inhibition rates are shown in Table I. The mean tumor weight in the EAEHJ treatment group was significantly lower than that of the control group $(\mathrm{P}<0.05)$. The inhibitory effects of EAEHJ treatment on tumor growth were evaluated from the tumor inhibition rates, the results of which indicated that EAEHJ suppressed tumor growth by $39.2 \%$ (Table I). However, administration of EAEHJ had no apparent effect on the body weight of the experimental animals, demonstrating that EAEHJ is able to inhibit liver cancer growth in vivo in the absence of apparent adverse effects. These results suggest that EAEHJ may significantly inhibit tumor growth in H22 tumor cell-bearing mouse model in vivo.

In order to examine the cytotoxicity and biological effects of EAEHJ, HepG2 human hepatoma cells were treated with various doses of EAEHJ $(0,0.5,0.75,1.5$ and $3.0 \mathrm{mg} / \mathrm{ml})$ for $24 \mathrm{~h}$. Any cellular morphological changes were subsequently evaluated by phase-contrast microscopy. As shown in Fig. 1, the control cells exhibited a typical polygonal intact shape, whereas the cells treated with various concentrations of EAEHJ for $24 \mathrm{~h}$ became rounded, shrunken, exhibited membrane blebbing, or became non-confluent. Furthermore, the effects of the various concentrations of EAEHJ on HepG2 human hepatoma cell viability were examined using an MTT assay. The results were concordant with the microscopy results indicating that EAEHJ inhibited cell growth in a dose-dependent manner. At a concentration of $0.5 \mathrm{mg} / \mathrm{ml}$, a significant loss of cell viability was observed. The cell growth inhibition rate was $\sim 50 \%$ at $0.75 \mathrm{mg} / \mathrm{ml}$ EAEHJ $(\mathrm{P}<0.01)$. Upon incubation with $3.0 \mathrm{mg} / \mathrm{ml}$ EAEHJ, the cells were only $40 \%$ viable, $(\mathrm{P}<0.01$, Fig. 1$)$. These results indicate that EAEHJ is able to inhibit the growth of HepG2 human hepatoma cells in vitro.

Effects of EAEHJ on cell apoptosis in the H22-bearing mice and HepG2 human hepatoma cells. In order to determine whether the inhibitory effects of EAEHJ on tumor growth were due to apoptosis, the present study examined the EAEHJ pro-apoptotic activities in tumor-bearing mice via IHC TUNEL staining. The percentage of TUNEL-positive cells in the control and EAEHJ-treated mouse groups was $31.5 \pm 11$ and $57.3 \pm 15 \%$, respectively $(\mathrm{P}<0.01$, Fig. $2 \mathrm{~A}$ and $\mathrm{B})$. These results indicate that EAEHJ may promote cell apoptosis in vivo.

The induction of cell apoptosis was evidenced by phosphatidylserine externalization in vitro. Annexin V-/ PI staining was performed in order to examine the occurrence of phosphatidylserine externalization on the cell surface. The number of Annexin V-stained cells, indicating both early and late 
A

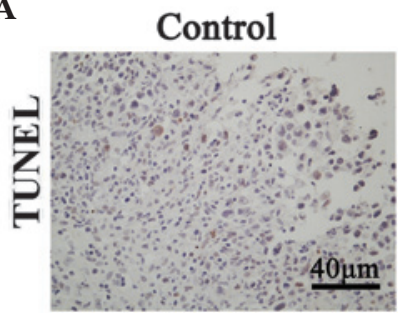

EAEHJ

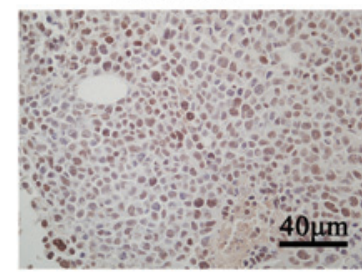

B

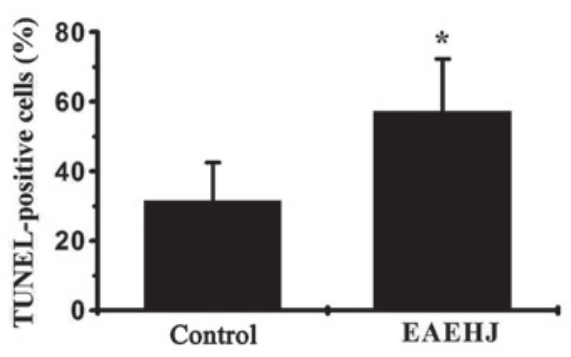

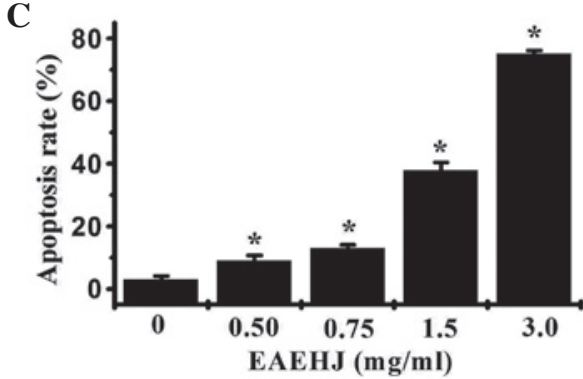

Figure 2. Effects of Hypericum japonicum ethyl acetate extract (EAEHJ) on cell apoptosis in a H22-bearing liver cancer mouse model. (A) At the end of the experiment, the tumor tissue samples from both the control and EAEHJ-treated groups were processed for immunohistochemical (IHC) staining using terminal deoxynucleotidyl transferase dUTP nick end labeling (TUNEL). Representative images were captured at a x400 magnification. (B) Quantification of the IHC assay was measured as the percentage of positively-stained cells. Data are presented as the mean \pm standard deviation ( $\mathrm{n}=8$ ). $\mathrm{P}<0.01$, vs. control. (C) Effects of EAEHJ on the apoptosis of HepG2 human hepatoma cells. HepG2 human hepatoma cells were collected and stained with Annexin V/propidium iodine followed by fluorescence-activated cell sorting analysis. The data are presented as the mean \pm standard deviation from three independent experiments. ${ }^{*} \mathrm{P}<0.01$, vs. the control cells.

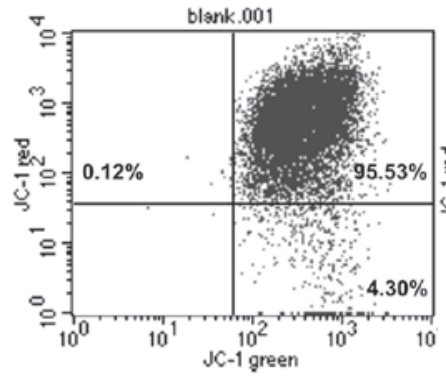

EAEHJ (0 mg/ml)

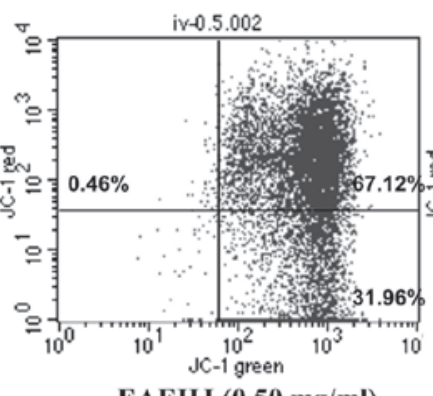

EAEHJ (0.50 mg/ml)

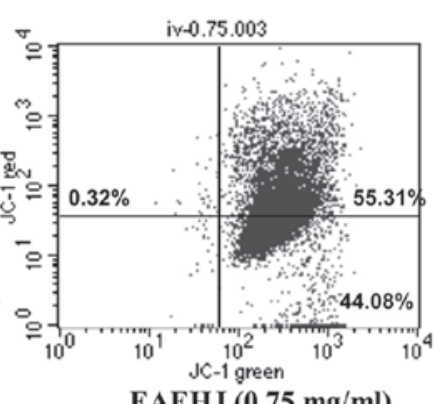

EAEHJ $(0.75 \mathrm{mg} / \mathrm{ml})$
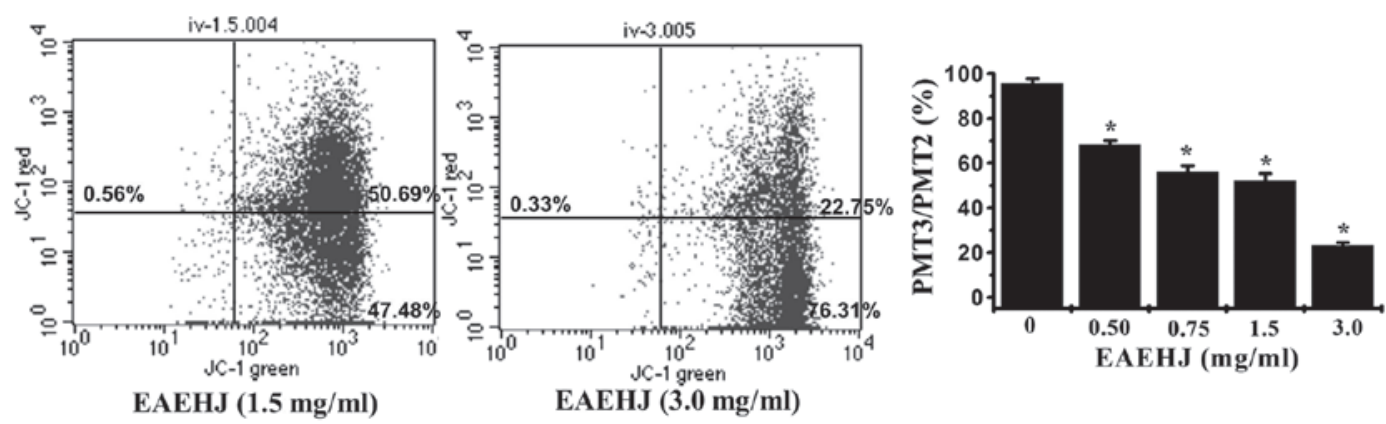

Figure 3. Effects of Hypericum japonicum ethyl acetate extract (EAEHJ) on the loss of mitochondrial membrane potential in HepG2 human hepatoma cells. HepG2 human hepatoma cells were treated with various concentrations of EAEHJ for 24 h, prior to being stained with JC-1. The mean JC-1 fluorescence intensity was detected using fluorescence-activated cell sorting analysis. The data are presented as the mean \pm standard deviation from three independent experiments, ${ }^{*} \mathrm{P}<0.01$, vs. the control cells.

apoptosis, increased with the concentration of EAEHJ treatment (Fig. 2C). At a low concentration of EAEHJ $(0.5 \mathrm{mg} / \mathrm{ml})$, only a small number of cells exhibited early-phase and late-phase apoptosis $(9.1 \%, \mathrm{P}<0.01)$. However, following incubation with higher concentrations of EAEHJ $(3.0 \mathrm{mg} / \mathrm{ml})$, the number of both early- and late-phase (necrotic stage) apoptotic cells increased
(75\%, $\mathrm{P}<0.01)$. These results indicate that cell treatment with EAEHJ may induce cell apoptosis in a dose-dependent manner.

Effects of EAEHJ on the loss of $\Delta \Psi m$. Early-stage cellular apoptosis is accompanied by disruption of the mitochondrial membrane, which leads to a rapid collapse of the electrochemical 
A
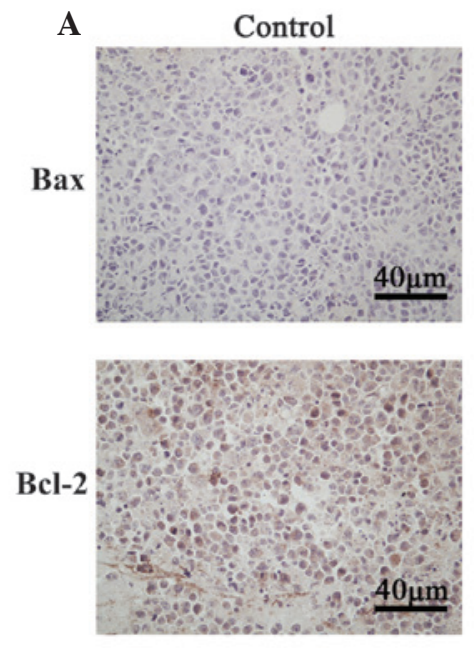

B

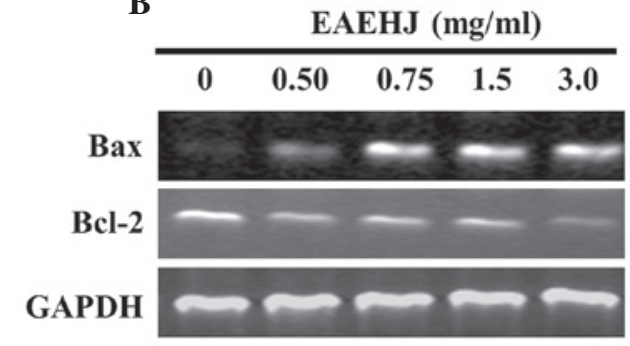

EAEHJ
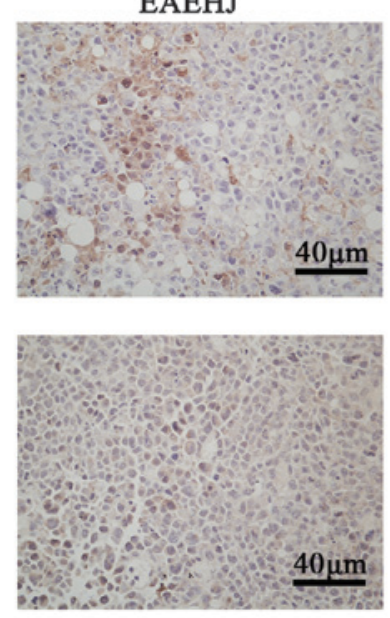

C
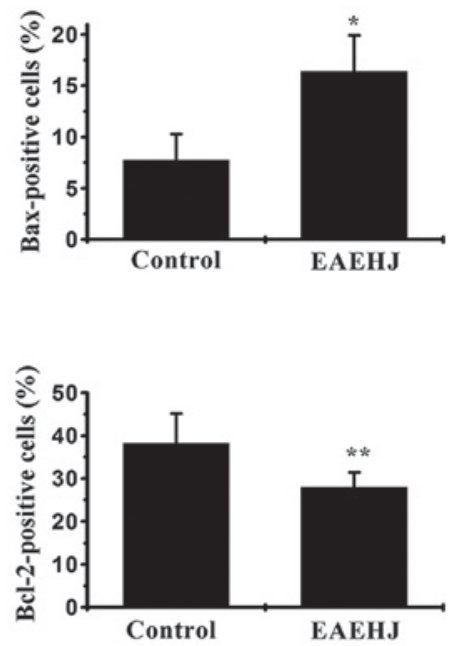

EAEHJ $(\mathrm{mg} / \mathrm{ml})$

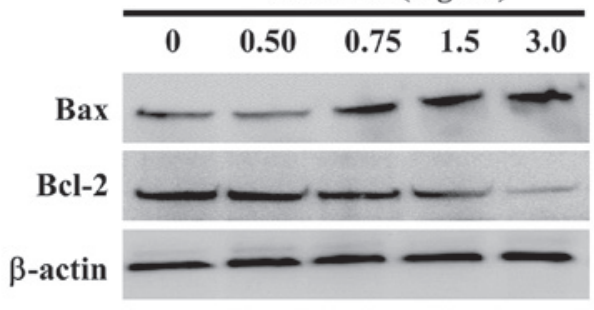

Figure 4. (A) Effects of Hypericum japonicum ethyl acetate extract (EAEHJ) on the expression levels of B-cell lymphoma 2 (Bcl-2) and Bcl-2-associated X protein (Bax) in a H22 cell-bearing liver cancer mouse model. At the end of the experiment, tumor tissue samples from the control and EAEHJ-treated groups were processed for immunohistochemical (IHC) staining in order to determine the expression levels of Bax and Bcl-2. Representative images were captured at a X400 magnification. Quantification of the IHC assay was measured as the percentage of positively-stained cells. The data are presented as the mean \pm standard deviation $(\mathrm{n}=8)$. ${ }^{*} \mathrm{P}<0.01$, and ${ }^{* *} \mathrm{P}<0.05$, vs. the control. (B and C) Effects of EAEHJ on the expression levels of Bax and Bcl-2 in HepG2 human hepatoma cells. HepG2 human hepatoma cells were treated with various concentrations of EAEHJ for 24 h. (B) The mRNA expression levels of Bax and Bcl-2 in the EAEHJ-treated and untreated cells were determined by reverse transcription-polymerase chain reaction (RTq-PCR). (C) The protein expression levels of Bax and $\mathrm{Bcl}-2$ were analyzed by western blotting. GAPDH and $\beta$-actin were used as internal controls for the RTq-PCR and western blotting assays, respectively. The data are representative of three independent experiments.

A

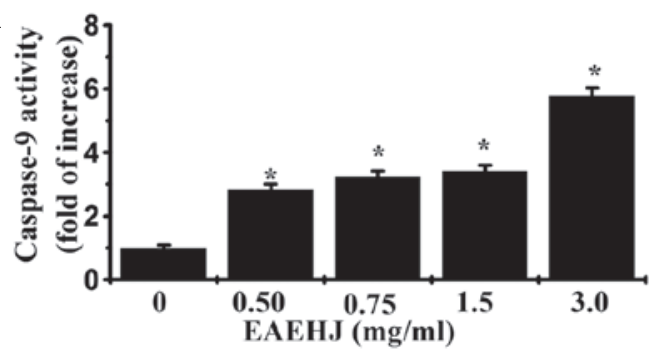

B

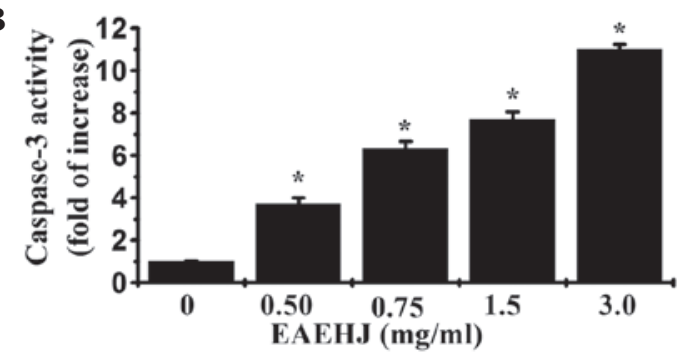

Figure 5. Effects of Hypericum japonicum ethyl acetate extract (EAEHJ) on the activity of caspases-3 and 9 in HepG2 human hepatoma cells. HepG2 human hepatoma cells were treated with various concentrations of EAEHJ for $24 \mathrm{~h}$. (A) Caspase-9 and (B) caspase-3 activity levels were determined using a colorimetric assay. The data were normalized to the caspase activities within the control cells (treated with $0.5 \%$ dimethyl sulfoxide vehicle) and represented as the "fold of control". The data are presented as the mean \pm standard deviation from three independent experiments. *P<0.01, vs. the control cells.

gradient. In the present study, the effects of EAEHJ on $\Delta \Psi \mathrm{m}$ were investigated using JC-1, a mitochondria-specific dye. JC-1 is a lipophilic, cationic dye that selectively enters mitochondria. In healthy cells with high $\Delta \Psi \mathrm{m}, \mathrm{JC}-1$ forms J-aggregates that exhibit intense red fluorescence (detected in PMT3, $590 \mathrm{~nm}$ ), whereas under apoptotic conditions, the $\Delta \Psi$ m collapses, and JC-1 does not accumulate within the mitochondria, but remains instead in the cytoplasm in its monomeric form, emitting green fluorescence (detected in PMT2, $525 \mathrm{~nm}$ ). Flow cytometric analysis demonstrated the loss of $\Delta \Psi \mathrm{m}$ in cells treated with
EAEHJ for $24 \mathrm{~h}$ in a dose-dependent manner. As shown in Fig. 3, following treatment with $0,0.5,0.75,1.5$, and $3.0 \mathrm{mg} / \mathrm{ml}$ of EAEHJ, the ratio of PMT3 to PMT2 was $95.8 \%, 68.2 \%, 56 \%$, $52.2 \%$, and $23.3 \%$, respectively $(\mathrm{P}<0.01)$. This dose-dependent change in $\Delta \Psi \mathrm{m}$ indicates that EAEHJ promotes the loss of $\Delta \Psi \mathrm{m}$ in HepG2 human hepatoma cells.

Effects of EAEHJ on the expression levels of Bax and Bcl-2 in H22 cell-bearing mice and HepG2 human hepatoma cell line. Pro-apoptotic Bax and anti-apoptotic Bcl-2 are two members 
of the Bcl-2 family that regulate the mitochondrial signaling pathway during apoptosis. Mitochondria-dependent apoptosis is primarily regulated by $\mathrm{Bcl}-2$ family proteins. It has been suggested that mitochondrial outer membrane permeabilization (MOMP) occurs through the formation of mitochondrial pores by pro-apoptotic Bax-like proteins, which may be inhibited by anti-apoptotic Bcl-2 like members $(14,15)$. Therefore, the ratio of Bax to Bcl-2 is crucial for determining cell fate. Higher Bcl-2 to Bax ratios, caused by the overexpression of Bcl-2 and/or downregulation of Bax, are commonly detected in various types of cancer (16), which confers a survival advantage to the cancer cells, and is associated with both chemotherapy and radiotherapy resistance. The results of the IHC assay indicated that treatment with EAEHJ reduced the expression levels of anti-apoptotic Bcl-2 in tumors, whereas the expression of pro-apoptotic Bax was increased. As shown in Fig. 4A, the percentage of Baxand Bcl-2-positive cells in the control group was 7.8 \pm 2.5 and $38.2 \pm 6.9 \%$, respectively, whereas those of the EAEHJ-treated mice were $16.4 \pm 3.5(\mathrm{P}<0.01$, vs. controls $)$ and $28 \pm 9.4 \%(\mathrm{P}<0.05$, vs. controls), respectively. These results indicate that treatment with EAEHJ may increase the pro-apoptotic Bax/Bcl-2 ratio. To our knowledge, this is the first time that EAEHJ has been reported to inhibit hepatoma cancer growth in vivo via the promotion of cancer cell apoptosis.

In order to further study the mechanism underlying the anti-carcinogenic effects of EAEHJ, RT-PCR and western blotting were performed to examine the mRNA and protein expression levels of Bax and Bcl-2 in the EAEHJ-treated HepG2 human hepatoma cells. The results of the RT-PCR assay demonstrated that treatment with EAEHJ significantly increased the mRNA expression levels of Bax and reduced the mRNA expression levels of Bcl-2 in the HepG2 human hepatoma cells (Fig. 4B). The results from the western blot analysis showed that the protein expression levels of Bax and Bcl-2 were similar to their respective mRNA expression levels, suggesting that EAEHJ induces mitochondria-dependent apoptosis of HepG2 human hepatoma cells through regulation of the expression levels of Bcl-2 family proteins (Fig. 4C).

Effects of EAEHJ on the activation of caspases-3 and 9. Caspases form part of the cysteine protease family, and are important proteins in the modulation of the apoptotic response. Caspase-3 is a key regulator of apoptosis, and is activated by an initiator caspase, such as caspase-9, during mitochondria-mediated apoptosis (17). In order to identify the downstream effectors of the apoptotic signaling pathway, the activation of caspase- 3 and 9 was examined using a colorimetric assay with the following caspase-specific chromophores: DEVD-pNA, a specific substrate of caspase-3, and LEHD-pNA, a specific substrate of caspase-9. As shown in Figs. 5A and B, treatment with EAEHJ significantly and dose-dependently induced activation of both caspase- 3 and 9 in the HepG2 human hepatoma cells $(\mathrm{P}<0.01)$, as compared with the untreated control cells. These results suggest that EAEHJ is able to promote HepG2 human hepatoma cell apoptosis via the mitochondria-dependent pathway.

\section{Discussion}

Natural products, specifically Chinese herbal medicines, have become increasingly important for their potency against various types of cancer, whilst having the advantage of fewer side effects and reduced drug resistance, as compared with conventional chemotherapy and radiotherapy. In the present study, using the H22 cell-bearing mouse model, it was demonstrated that Hypericum japonicum may inhibit cancer growth in vivo, with no apparent effect on murine body weight, indicating that it had no apparent toxicity.

Apoptotic mechanisms are commonly exploited for tumor therapy. Apoptotic cell death is recognizable by cellular morphological characteristics, including membrane blebbing and chromatin condensation (18). As demonstrated by the current study, the control cells exhibited a typical polygonal intact shape, whereas the cells treated with various concentrations of Hypericum japonicum, became rounded, shrunken, exhibited membrane blebbing, or became non-confluent.

The initiation of apoptosis involves two major signaling pathways: Extrinsic (death receptor) and intrinsic (mitochondrial). The intrinsic apoptotic pathway is regulated by mitochondria which has an central role in apoptosis (19). In the early stages of apoptosis, the membrane phosphatidylserine translocates from the inner side of the plasma membrane to the outer layer, and becomes exposed at the external surface of the cell (20). Annexin $\mathrm{V}$ is a $\mathrm{Ca}^{2+}$ dependent phospholipid-binding protein with high affinity for phosphatidylserine (19). Using Annexin-V/PI staining followed by FACS analysis it was demonstrated that Hypericum japonicum treatment was able to significantly induce the apoptosis of HepG2 human hepatoma cells. MOMP is the point of convergence for a large variety of intracellular apoptotic signals leading to the release of numerous apoptogenic proteins from the mitochondrial intermembrane space (21). Loss of mitochondrial membrane potential $(\Delta \Psi \mathrm{m})$ has been suggested to contribute to cell death by disruption of normal mitochondrial function $(22,23)$. The current study investigated whether Hypericum japonicum is able to promote the deregulation of $\Delta \Psi \mathrm{m}$ in HepG2 human hepatoma cells.

The Bcl-2 family of proteins are important regulators of apoptosis, including pro-apoptotic members such as Bax and anti-apoptotic members such as Bcl-2 (24,25). The ratio of active anti-apoptotic Bax to pro-apoptotic Bcl-2 determines cellular fate, and variations in this ratio results in impaired apoptosis, leading to various diseases, including cancer (26-28). RT-PCR, western blotting and IHC were conducted to examine the mRNA and protein expression levels of the pro-apoptotic protein Bax and the anti-apoptotic protein Bcl-2 in the Hypericum japonicum-treated human hepatoma cells. It was demonstrated that Hypericum japonicum treatment dose-dependently enhanced Bax expression and reduces Bcl-2 expression in at mRNA and protein levels.

Caspases, a family of cysteine proteases, are key proteins that modulate the apoptotic response, and caspase- 3 is a key executioner of apoptosis $(29,30)$. In the current study, it was demonstrated that the induction of apoptosis may involve the loss of mitochondrial transmembrane potential, and caspase- 9 and caspase- 3 activation.

In conclusion, to the best of our knowledge for the first time, it is reported that Hypericum japonicum is able to significantly inhibit the growth of H22-cell tumors without affecting murine weight, indicating that Hypericum japonicum is non-toxic with few side effects. The results of the present study additionally 
demonstrated that Hypericum japonicum promotes cancer cell apoptosis via the mitochondria-dependent pathway in vitro. These results may help establish a scientific foundation for future research and therapeutic development of EAEHJ as an effective anti-carcinogenic agent.

\section{Acknowledgements}

The authors of the present study are grateful to Dr DaWo (Tongji University School of Medicine, Shanghai, China) for reviewing the manuscript of the present study, and to Dr Zhenyu Zhang (Tongji University School of Medicine) for editing the figures of the present study. The present study was supported by grants from the Open Foundation of Fujian Key Laboratory of Integrative Medicine on Geriatrics (grant no. ZXY2008003), the Development Foundation of Chen Keji Integrative Medicine (grant no. CKJ2008007), the Education Department of Fujian Province (grant no. JA10169), the Health Department of Fujian Province (grant no. 2010-1-39), and the Open Foundation of Fujian Health College (grant no. 2010-2-10).

\section{References}

1. Jemal A, Bray F, Center MM, Ferlay J, Ward E and Forman D: Global cancer statistics. CA Cancer J Clin 61: 69-90, 2011.

2. Ferlay J, Shin HR, Bray F, Forman D, Mathers C and Parkin DM: Estimates of worldwide burden of cancer in 2008: GLOBOCAN 2008. Int J Cancer 127: 2893-2917, 2010.

3. Wang N, Li P, Wang Y, Peng W, Wu Z, Tan S, Liang S, Shen X and Su W: Hepatoprotective effect of Hypericum japonicum extract and its fractions. J Ethnopharmacol 116: 1-6, 2008.

4. Wang XW, Zhang DW, Wei XL, et al: Advances in research on Hypericum japonicum. Chin J Mod Drug Appl 3: 183-185, 2009 (In Chinese).

5. Shen YZ, Zhang XC and Tang WZ: Advances in research on Hypericum japonicum of its chemical components and pharmacological activities. Shangdong Pharm Ind 22: 28-29, 2003 (In Chinese).

6. Sun ZY, Jin GJ, Xu T, et al: Analysis of 30 patients of Primary Liver Cancer treated by Hypericum japonicum thunb. Chin J Integr Trad Western Med Liver Dis 5: 29-30, 1995 (In Chinese).

7. Li QX, Peng RX and Gao P: Hepatoprotective effect of Tianjihuang injection against APAP-induced hepatic toxicity in mice. Chin Pharm J 27: 472-474, 1992 (In Chinese).

8. Dong J, Liu Y, Liang Z and Wang W: Investigation on ultrasound-assisted extraction of salvianolic acid B from Salvia miltiorrhiza root. Ultrason Sonochem 17: 61-65, 2010.

9. Guide for the Care and Use of Laboratory Animals. National Research Council (US) Institute of Animal Research, National Academies Press (US), Washington (DC), USA, 1996.

10. Chang A, Cai Z, Wang Z and Sun S: Extraction and isolation of alkaloids of Sophora alopecuroides and their anti-tumor effects in H22 tumor-bearing mice. Afr J Tradit Complement Altern Med 11: 245-248, 2014.

11. Gao L, Chen L, Fei XH, Qiu HY, Zhou H and Wang JM: STI571 combined with vincristine greatly suppressed the tumor formation of multidrug-resistant K562 cells in a human-nude mice xenograft model. Chin Med J (Engl) 119: 911-918, 2006.

12. Jin Y, Li J, Rong LF, Li YH, Guo L and Xu SY: Anti-hepatocarcinoma effects of 5-fluorouracil encapsulated by galactosylceramide liposomes in vivo and in vitro. World J Gastroenterol 11: 2643-2646, 2005.
13. Chen H, Takahashi S, Imamura M, Okutani E, Zhang ZG, Chayama $\mathrm{K}$ and Chen BA: Earthworm fibrinolytic enzyme: Anti-tumor activity on human hepatoma cells in vitro and in vivo. Chin Med J (Engl) 120: 898-904, 2007.

14. Chipuk JE and Green DR: How do BCL-2 proteins induce mitochondrial outer membrane permeabilization? Trends Cell Biol 18: 157-164, 2008.

15. Arnoult D: Apoptosis-associated mitochondrial outer membrane permeabilization assays. Methods 44: 229-234, 2008.

16. Souers AJ, Leverson JD, Boghaert ER, Ackler SL, Catron ND, Chen J, Dayton BD, Ding H, Enschede SH, Fairbrother WJ, et al: ABT-199, a potent and selective BCL-2 inhibitor, achieves antitumor activity while sparing platelets. Nat Med 19: 202-208, 2013.

17. Ma Y, Zhang A, Shi Z, He C, Ding J, Wang X, Ma J and Zhang H: A mitochondria-mediated apoptotic pathway induced by deoxynivalenol in human colon cancer cells. Toxicol In Vitro 26: 414-420, 2012.

18. Lütticken C, Wegenka UM, Yuan J, Buschmann J, Schindler C, Ziemiecki A, Harpur AG, Wilks AF, Yasukawa K, Taga T, et al: Association of transcription factor APRF and protein kinase Jak1 with the interleukin-6 signal transducer gp130. Science 263: 89-92, 1994.

19. Elmore S: Apoptosis: A review of programmed cell death. Toxicol Pathol 35: 495-516, 2007.

20. Kotwicka M, Filipiak K, Jedrzejczak P and Warchol JB: Caspase-3 activation and phosphatidylserine membrane translocation in human spermatozoa: is there a relationship? Reprod Biomed Online 16: 657-663, 2008.

21. David R: Apoptosis: A lipid trigger of MOMP. Nat Rev Mol Cell Biol 13: 208-209, 2012.

22. Ly JD, Grubb DR and Lawen A: The mitochondrial membrane potential (deltapsi(m)) in apoptosis; an update. Apoptosis 8: 115-128, 2003.

23. Sesso A: Pitfalls in the use of electron microscopy to study the mitochondrial membrane permeability transition in apoptotic cells and pellets: Where do we stand in relation to the incidence of mitochondrial swelling in apoptosis? Braz J Morphol Sci 23: 57-74, 2006.

24. Adams JM and Cory S: The Bcl-2 apoptotic switch in cancer development and therapy. Oncogene 26: 1324-1337, 2007.

25. Shamas-Din A, Kale J, Leber B and Andrews DW: Mechanisms of action of Bcl-2 family proteins. Cold Spring Harb Perspect Biol 5: a008714, 2013.

26. Bishayee K, Ghosh S, Mukherjee A, Sadhukhan R, Mondal J and Khuda-Bukhsh AR: Quercetin induces cytochrome- $c$ release and ROS accumulation to promote apoptosis and arrest the cell cycle in $\mathrm{G} 2 / \mathrm{M}$, in cervical carcinoma: Signal cascade and drug-DNA interaction. Cell Prolif 46: 153-163, 2013.

27. Smerage JB, Budd GT, Doyle GV, Brown M, Paoletti C, Muniz M, Miller MC, Repollet MI, Chianese DA, Connelly MC, et al: Monitoring apoptosis and $\mathrm{Bcl}-2$ on circulating tumor cells in patients with metastatic breast cancer. Mol Oncol 7: 680-692, 2013.

28. Thomas S, Quinn BA, Das SK, Dash R, Emdad L, Dasgupta S, Wang XY, Dent P, Reed JC, Pellecchia M, et al: Targeting the Bcl-2 family for cancer therapy. Expert Opin Ther Targets 17: 61-75, 2013.

29. Brentnall M, Rodriguez-Menocal L, De Guevara RL, Cepero E and Boise LH: Caspase-9, caspase-3 and caspase-7 have distinct roles during intrinsic apoptosis. BMC Cell Bio 14: 32, 2013.

30. Cohen GM: Caspases: The executioners of apoptosis. Biochem J 326: 1-16, 1997. 